\title{
Geranylgeranyl transferase 1 inhibitor GGTI-298 enhances the anticancer effect of gefitinib
}

\author{
BI-SHENG LIU ${ }^{1,2^{*}}$, XIN-YU DAI $^{1,2^{*}}$, HONG-WEI XIA ${ }^{2}$, HUAN-JI XU ${ }^{1,2}$, \\ QIU-LIN TANG ${ }^{2}$, QI-YONG GONG ${ }^{3}$, YONG-ZHAN NIE ${ }^{4}$ and FENG BI ${ }^{1,2}$
}

\begin{abstract}
${ }^{1}$ Department of Medical Oncology, Cancer Center, West China Hospital; ${ }^{2}$ Laboratory of Molecular Targeted Therapy in Oncology, State Key Laboratory of Biotherapy; ${ }^{3}$ Department of Radiology, West China Hospital, Sichuan University, Chengdu, Sichuan 610041; ${ }^{4}$ State Key Laboratory of Cancer Biology and Xijing Hospital of Digest Diseases, Fourth Military Medical University, Xi'an, Shaanxi 710032, P.R. China
\end{abstract}

Received January 9, 2018; Accepted May 11, 2018

DOI: $10.3892 / \mathrm{mmr} .2018 .9371$

\begin{abstract}
Dysregulation of epidermal growth factor receptor (EGFR) signaling is responsible for the resistance to EGFR tyrosine kinase inhibitors (TKIs), such as gefitinib and erlotinib, and is thereby associated with the progression of tumors in non-small cell lung cancers (NSCLCs). Immunoblotting results revealed that geranylgeranyl transferase 1 inhibitor (GGTI)-298, a geranylgeranyl transferase 1 inhibitor with potential antitumor effects, effectively inhibited the phosphorylation of EGFR and its downstream target protein kinase B (AKT). A combination of gefitinib and GGTI-298 amplified the inhibition of the EGFR-AKT signaling pathway. In addition, GGTI-298 treatment produced a synergistic effect on the inhibition of proliferation as indicated by the combination index values of $<1$ when combined with gefitinib in the NSCLC cell lines HCC827 and A549. These synergistic effects were also observed to induce apoptosis and migration inhibition. Further mechanistic studies demonstrated that GGTI-298 inhibited the activity of Ras homolog family member A (RhoA), and downregulation of RhoA with small interfering RNA impaired the phosphorylation of EGFR, which suggested that EGFR inhibition by GGTI-298 may be exerted mainly through RhoA mediation. These results presented a novel, promising therapeutic strategy involving a combination of two drugs for targeting EGFR signaling in lung cancer.
\end{abstract}

Correspondence to: Professor Feng Bi, Laboratory of Molecular Targeted Therapy in Oncology, State Key Laboratory of Biotherapy. West China Hospital, Sichuan University, 37 Wai Nan Guoxue Road, Chengdu, Sichuan 610041, P.R. China

E-mail: bifeng@scu.edu.cn

${ }^{*}$ Contributed equally

Key words: epidermal growth factor receptor, gefitinib, geranylgeranyl transferase 1 inhibitor-298, non-small cell lung cancer, Ras homolog family member A

\section{Introduction}

Tyrosine kinase inhibitors (TKIs), such as gefitinib and erlotinib, target epidermal growth factor receptors (EGFRs) and have been demonstrated to be efficacious in treating non-small cell lung cancers (NSCLCs) (1-3). The dysregulation of EGFR signaling is closely associated with cell proliferation and EGFR TKI resistance $(4,5)$, which highlights the importance of complete EGFR signaling inhibition in lung cancer treatment.

Geranylgeranylation is an important biochemical modification for cellular localization and the function of small G proteins $(6,7)$. Previous studies have reported that geranylgeranylation is critical for cancer cell proliferation and invasion $(8,9)$, which indicates the potential anticancer function of inhibiting geranylgeranylation. The geranylgeranyl transferase 1 (GGT1) inhibitor (GGTI)-298 inhibits protein geranylgeranylation. Recent studies in vitro and in animal models have revealed that GGTI-298 inhibited cell proliferation and induced apoptosis when applied as a single agent or in combination with chemotherapeutics (10-12). In addition, GGTIs inhibited EGF-mediated EGFR tyrosine phosphorylation, indicating that the GGT1 substrate may serve a vital role in the process of EGFR phosphorylation (13). However, the molecular mechanism underlying the GGTI-induced inhibition of EGFR phosphorylation has not been well studied.

Ras homolog family member A (RhoA), a widely studied protein in the Rho family of guanosine triphosphate (GTP)-ases, participates in various physiological processes, including cytoskeleton organization, cell cycle progression, cell survival and cell migration (14). A growing number of studies have revealed that RhoA interacts with various signaling molecules to exert effects on cell proliferation and apoptosis (15-17). Thus, a deeper understanding of RhoA-associated signaling regulation will support the identification of novel strategies and modalities in cancer treatment.

In the present study, a potentially favorable interaction between GGTI-298 and the EGFR TKI gefitinib was identified. The two combined stimulated cytotoxicity and induced apoptosis in lung cancer cells. The synergistic mechanism was also studied. 


\section{Materials and methods}

Cell culture and agents. The NSCLC cell lines (HCC827, A549, H1975, SPCA1 and H1299) were purchased from American Type Culture Collection (Manassas, VA, USA). All cell lines were cultured in Dulbecco's modified Eagle's medium (Gibco; Thermo Fisher Scientific, Inc., Waltham, MA, USA) supplemented with or without $10 \%$ heat inactivated fetal bovine serum (Gibco; Thermo Fisher Scientific, Inc.) depending on the assay. Cell cultures were maintained at $37^{\circ} \mathrm{C}$ in a humidified (5\% $\mathrm{CO}_{2}, 95 \%$ air) incubator. GGTI-298 and gefitinib were purchased from Selleck Chemicals (Houston, TX, USA), and dimethylsulfoxide (DMSO) and EGF were purchased from Sigma-Aldrich; Merck KGaA (Darmstadt, Germany).

Immunoblotting analysis. A549 cells were treated with $5 \mu \mathrm{M}$ GGTI-298 in serum-free medium for $48 \mathrm{~h}$. Prior to protein extraction, the cultures were exposed to $30 \mathrm{ng}$ EGF for $30 \mathrm{~min}$. Cells (HCC827, A549, H1975, SPCA1 and H1299) were lysed with ice-cold Radioimmunoprecipitation Assay buffer [150 mM $\mathrm{NaCl}, 1 \% \mathrm{NP}-40,50 \mathrm{mM}$ Tris- $\mathrm{HCl}$ (pH 7.4), $1 \mathrm{mM}$ phenylmethylsulfonyl fluoride, $1 \mu \mathrm{g} / \mathrm{ml}$ leupeptin, $1 \mathrm{mM}$ deoxycholic acid and $1 \mathrm{mM}$ EDTA] containing a protease inhibitor cocktail (Sigma-Aldrich; Merck KGaA). The protein concentration was detected using the BCA assay (Thermo Fisher Scientific, Inc., Waltham, MA, USA). In total, $\sim 20 \mu \mathrm{g}$ total protein was loaded on to a $12 \%$ SDS-PAGE gel, separated electrophoretically and then transferred to nitrocellulose membranes (EMD Millipore, Billerica, MA, USA). The membranes were blocked with 5\% nonfat milk at room temperature for $30 \mathrm{~min}$ and were washed three times with phosphate buffer saline (PBS). The membranes were incubated with the following primary antibodies: Phosphorylated (p)-EGFR (cat. no. 3777), cyclin D1 (cat. no. 2922), phosphatase and tensin homolog (PTEN; cat. no. 9552), GAPDH (cat. no. 97166), glutathione S-transferase (GST; all Cell Signaling Technology, MA, USA), p-extracellular signal-regulated kinase (ERK; cat. no. EP197Y), ERK (cat. no. ab 17942), p-protein kinase B (AKT; cat. no. EP2109Y), AKT (cat. no. EPR16798; all Abcam, Cambridge, UK), EGFR (cat. no. sc-03-G), 3-hydroxy-3-methylglutaryl-co-enzyme A reductase (HMGCR; cat. no. sc-271595) and RhoA (cat. no. sc-418; all Santa Cruz Biotechnology, Inc., Dallas, TX, USA). All of the primary antibodies were added at a 1:1,000 dilution overnight at $4^{\circ} \mathrm{C}$. Subsequently, the membranes were incubated with the horseradish peroxidase conjugated anti-mouse (cat. no. HA1022) or anti-rabbit (cat. no. HA1001) secondary antibodies with dilution of 1:5,000 (Huabio, Hangzhou, China) at room temperature for $3 \mathrm{~h}$ and were washed with PBS. Protein expression was detected using the ODYSSEY Infrared Imaging System (LI-COR Biosciences, Lincoln, NE, USA) and quantified using Image J 1.48 (National institute of Health, Bethesda, MA, USA).

Cell proliferation assay. HCC827 and A549 cells were seeded $\left(\sim 5 \times 10^{3}\right.$ cells per well in $100 \mu 1$ DMEM with $10 \%$ FBS $)$ in a 96-well flat-bottomed plate (Corning Incorporated, Corning, NY, USA) at $24 \mathrm{~h}$ prior to treatment, namely, HCC827 and A549 cells were treated with gefitinib (HCC827, $10 \mathrm{nM}$; A549, $5 \mu \mathrm{M}$ ) and/or GGTI-298 (concentration indicated) for $48 \mathrm{~h}$ at $37^{\circ} \mathrm{C}$ in a humidified $\left(5 \% \mathrm{CO}_{2}, 95 \%\right.$ air) incubator.
Following incubation at $37^{\circ} \mathrm{C}$, cell growth was measured using a Cell Counting Kit-8 (Dojindo Molecular Technologies, Inc., Kumamoto, Japan). Synergistic activity between gefitinib and GGTI-298 was assessed using combination index (CI) analysis, where a $\mathrm{CI}<1$ indicated synergism, $\mathrm{CI}=1$ indicated additive effects and CI $>1$ indicated antagonism (18).

Cell apoptosis assay. HCC827 and A549 cells were seeded $\left(\sim 5 \times 10^{3}\right.$ cells per well in $100 \mu 1$ medium $)$ in a 6 -well flat-bottomed plate $24 \mathrm{~h}$ prior to treatment, namely, HCC 827 and A549 cells were treated with DMSO, gefitinib (HCC827, $10 \mathrm{nM}$; A549, $5 \mu \mathrm{M}$ ), GGTI-298 (HCC 827, $5 \mu \mathrm{M}$; A549, $7.5 \mu \mathrm{M}$ ) or a combination of gefitinib and GGTI-298 for $48 \mathrm{~h}$ at $37^{\circ} \mathrm{C}$ in a humidified $\left(5 \% \mathrm{CO}_{2}, 95 \%\right.$ air) incubator. Apoptotic cells were detected using Hoechst 33342 (Sigma-Aldrich; Merck KGaA) DNA staining (room temperature for $10 \mathrm{~min}$ ), according to the kit instructions. Apoptotic cells were characterized by nuclear condensation and DNA fragmentation, which were observed by fluorescence microscopy (Nikon Corporation, Tokyo, Japan).

Wound healing assay. HCC 827 and A549 cells $\left(\sim 5 \times 10^{5}\right)$ were seeded in a 6-well culture plate for $24 \mathrm{~h}$ at $37^{\circ} \mathrm{C}$. Wounding was performed by scraping through the cell monolayer with a $200 \mu 1$ pipette tip. Medium and nonadherent cells were removed and replaced with $2 \mathrm{ml}$ of fresh serum-free medium containing the indicated reagents (DMSO, gefitinib, GGTI-298 or a combination of gefitinib and GGTI-298). Cells were permitted to migrate for $48 \mathrm{~h}$ at $37^{\circ} \mathrm{C}$. Wound healing was photographed microscopically (light) at 0,24 and $48 \mathrm{~h}$. Three fields of view for each wound were randomly selected, and the average wound width was calculated. The relative wound width was calculated as follows: wound width at the indicated time $(0,24$ or $48 \mathrm{~h}) /$ wound width at $0 \mathrm{~h}$.

Pull-down assays. Briefly, to detect the levels of active RhoA, equal amounts $\left(\sim 8 \times 10^{6}\right.$ cells) of A549 cell lysates $[20 \mathrm{mM}$ Hepes, $100 \mathrm{mM} \mathrm{NaCl}, 0.5 \%$ NP-40, $10 \%$ glycerol, $0.2 \%$ deoxycholic acid, and $10 \mathrm{mM} \mathrm{MgCl}{ }_{2},(\mathrm{pH}$ 7.5)] were incubated with GST-Rho binding domain proteins immobilized on glutathione-Sepharose beads at $4^{\circ} \mathrm{C}$ overnight. The beads were then washed three times with ice-cold phosphate buffered solution. Bound proteins were eluted from the beads with SDS-PAGE sample buffer. RhoA protein was then analyzed by immunoblotting with anti-RhoA antibody, which was performed as previously described.

Transfection. Small interfering (si)-RNAs against RhoA and the control were synthesized by Shanghai GenePharma Co., Ltd. (Shanghai, China). The sequence of the RhoA siRNA was 5'-GACAUGCUUGCUCAUAGUCTT-3', and the sequence of the control siRNA was 5'-AAUUCUCCGAACGUGUCACGU UU-3'. A549 cells were transfected with $50 \mathrm{nM}$ control siRNA and RhoA siRNA for $72 \mathrm{~h}$ using Lipofectamine $2000^{\mathrm{TM}}$ (Invitrogen; Thermo Fisher Scientific, Inc.) according to the manufacturer's instructions.

Statistical analysis. All statistical analyses were performed with SPSS 14.0 for Windows software (SPSS, Inc., Chicago, IL, USA). The results were analyzed using the Student's t-test 

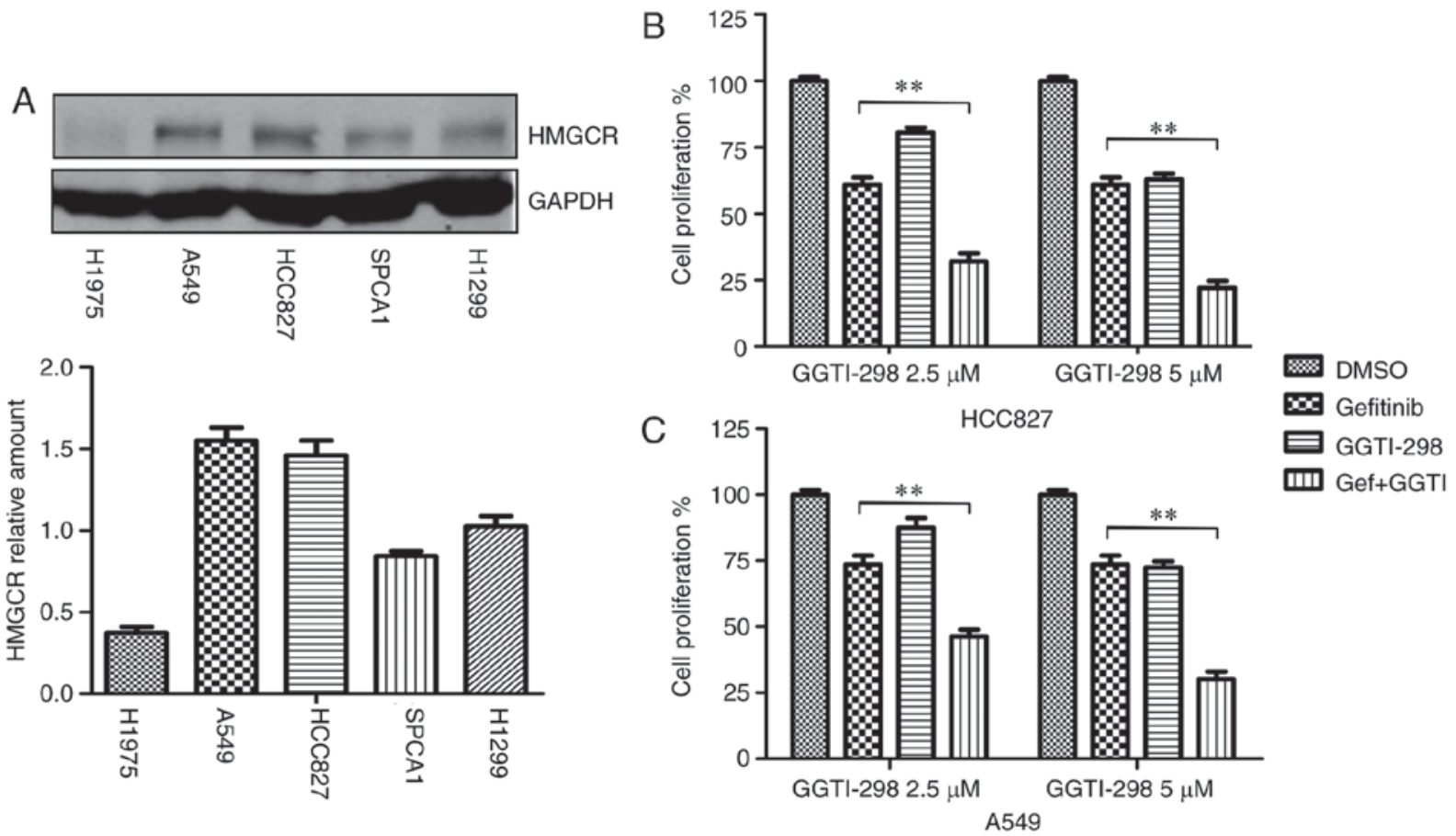

Figure 1. Synergic antiproliferation effect of gefitinib and GGTI-298 in NSCLC cell lines. (A) The relative protein expression of HMGCR compared with GAPDH in each of the five NSCLC cell lines following immunoblotting analysis. GAPDH was used as an internal control; the relative amount of HMGCR was calculated as, HMGCR expression/GAPDH expression in each cell line, with the relative HMGCR expression of H1299 cells set as 1. Cell proliferation was measured by Cell Counting Kit-8 in (B) HCC827 and (C) A549 cells treated with gefitinib (HCC827, 10 nM; A549, $5 \mu$ M) and/or GGTI-298 (concentration indicated) for $48 \mathrm{~h}$. The results were presented as the mean \pm standard error of the mean ( $\mathrm{n}=3$ biological replicates). ${ }^{* *} \mathrm{P}<0.01$, as indicated. HMGCR, 3-hydroxy-3-methylglutaryl-co-enzyme A reductase; GGTI-298, geranylgeranyl transferase 1 inhibitor; NSCLC, non-small cell lung cancer; DMSO, dimethylsulfoxide.

when two groups were compared, or by one-way analysis of variance with Tukey's post hoc test for multiple comparisons. $\mathrm{P}<0.05$ was considered to indicated a statistically significant difference. Values are presented as the mean \pm standard error of the mean.

\section{Results}

Synergic antiproliferation effect of gefitinib and GGTI-298 in NSCLC cell lines A549 and HCC827. As GGTI-298 is an inhibitor of a branch of the mevalonate pathway (19), the present study first detected the relative protein expression of HMGCR (the rate-limiting enzyme of mevalonate pathway) in five cell lines (H1975, A549, HCC827, SPCA1 and H1299) and then the two cell lines with a relatively high expression of HMGCR, HCC827 and A549, were selected for further experimentation (Fig. 1A). To determine the effect of GGTI-298 on gefitinib sensitivity, lung cancer cells were treated with gefitinib, GGTI-298 and combination of gefitinib and GGTI-298. The results revealed that the combined treatment of gefitinib and GGTI-298 inhibited cell proliferation more thoroughly than either of the single drug treatments, in a dose-dependent manner (Fig. 1B and C). The CI values indicated synergy, with values of 0.48 and 0.58 for HCC827 and A549 cells, respectively.

Enhanced effects of gefitinib and GGTI-298 on the apoptosis and migration of lung cancer cells. To investigate the effect of GGTI-298 on gefitinib-induced apoptosis, Hoechst assays were conducted. The results demonstrated that a combination of GGTI-298 and gefitinib markedly promoted cell apoptosis when compared with each monotherapy group (Fig. 2A and B). Addition of GGTI-298 increased apoptosis by $>25 \%$ when compared with gefitinib alone (percentage of apoptotic cells: HCC827, gefitinib vs. gefitinib+GGTI-298=18.3 vs. $48.3 \%$, $\mathrm{P}<0.01$; A549, gefitinib vs. gefitinib+GGTI-298=12 vs. $39.7 \%$, $\mathrm{P}<0.01$; Fig. 2B). Then, wound healing assays were conducted to investigate the influence of GGTI-298 and gefitinib on cell migration. The results revealed that migration ability was significantly inhibited with a combination of gefitinib and GGTI-298 when compared with either single drug treatment following $48 \mathrm{~h}$ (Fig. 2C and D). The relative wound widths (wound width at the indicated time/wound width at $0 \mathrm{~h}$ ) at $48 \mathrm{~h}$ of gefitinib and gefitinib+GGTI-298 were 57.5 and $89.0 \%$ in HCC827 cells, and 53.5 and $83.6 \%$ in A549 cells, respectively $(\mathrm{P}<0.05$; Fig. 2D).

Effects of gefitinib and GGTI-298 on the EGFR signaling pathway and cyclin D1. To gain a greater understanding of the mechanism underlying the combined effect of GGTI-298 and gefitinib, the present study evaluated protein expression by performing immunoblotting. Protein expression was examined following $48 \mathrm{~h}$ of treatment with gefitinib and/or GGTI-298. As shown in Fig. 3, GGTI-298 treatment effectively inhibited the expression of pEGFR and pAKT. Furthermore, inhibition of the EGFR-AKT signaling pathway was substantially amplified when GGTI-298 and gefitinib were combined (Fig. 3). However, the present study did not observe marked changes 
A

A
HCC827

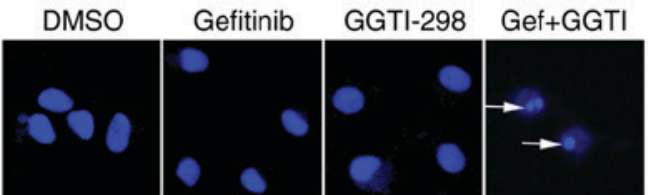

A549

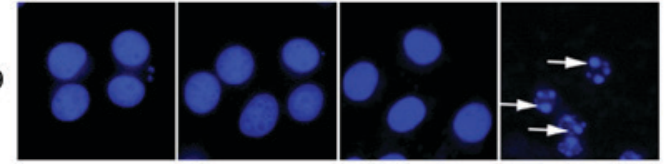

C

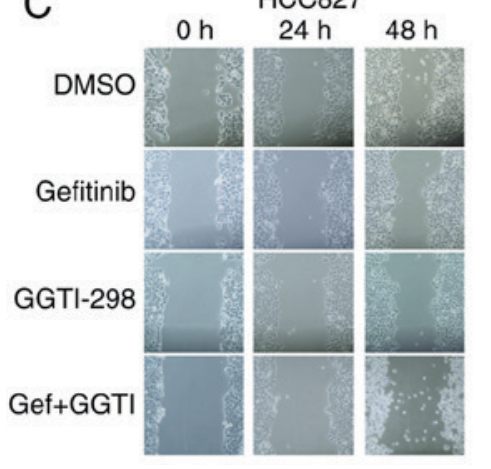

B

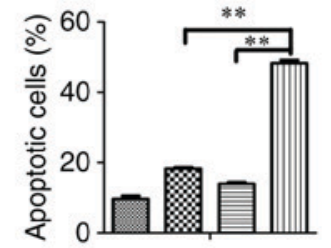

HCC 827

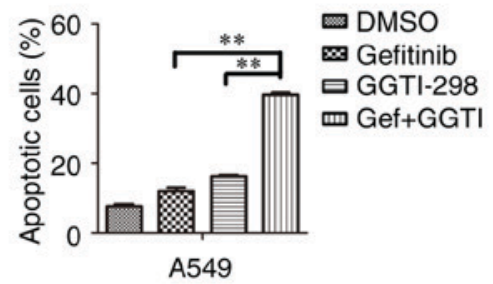

D

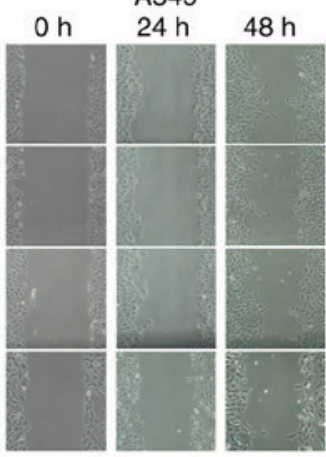

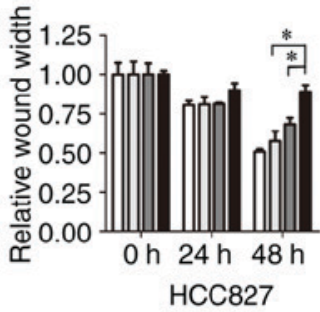

Figure 2. Synergistic effects of gefitinib and GGTI-298 on apoptosis and migration. (A) Cell apoptosis was measured by Hoechst staining and (B) the number of apoptotic cells was recorded. HCC827 and A549 cells were treated with DMSO, gefitinib (HCC827, $10 \mathrm{nM}$; A549, $5 \mu$ M), GGTI-298 (HCC827, $5 \mu$ M; A549, $7.5 \mu \mathrm{M})$ or a combination of gefitinib and GGTI-298 for $48 \mathrm{~h}$. Representative images of immunofluorescence are presented. Apoptotic cells are indicated by white arrows (magnification, $\mathrm{x} 400$ ). At least 300 cells were scored in every replicate. (C) Cell migration was detected using wound healing assays for HCC 827 and A549 cells treated with DMSO, gefitinib (HCC827, $10 \mathrm{nM}$; A549, $5 \mu \mathrm{M}$ ), $5 \mu \mathrm{M}$ GGTI-298 or a combination of gefitinib and GGTI-298 for 48 h. (D) The ratio of the relative wound width was calculated as follows: wound width at the indicated time $(0,24 \mathrm{or} 48 \mathrm{~h}) /$ wound width at $0 \mathrm{~h}$. Representative images from the wound healing assays are shown. The results were presented as the mean \pm standard error of the mean ( $\mathrm{n}=3$ biological replicates). ${ }^{*} \mathrm{P}<0.05$ and ${ }^{* *} \mathrm{P}<0.01$, as indicated. GGTI/GGTI-298, geranylgeranyl transferase 1 inhibitor; Gef, gefitinib; DMSO, dimethylsulfoxide.

in pERK expression with GGTI-298 treatment alone (Fig. 3). The protein expression of cyclin D1 was also analyzed, which is a proliferation-associated protein and a downstream target of AKT (20). Consistent with the pEGFR and pAKT results, treatment with gefitinib and GGTI-298 induced additional cyclin D1 downregulation when compared with either monotherapy (Fig. 3).

GGTI-298 inhibits the EGFR-AKT signaling pathway through RhoA. The present study then examined the specific mechanism underlying pAKT downregulation. Immunoblotting was employed to analyze the expression of pEGFR (which is expressed upstream of pAKT), PTEN (a negative regulator of $\mathrm{pAKT}$ ) and pAKT. The results revealed that pEGFR and pAKT expression increased markedly following treatment with EGF, while GGTI-298 treatment reversed the activation of pEGFR and pAKT signaling induced by EGF (Fig. 4A). However, PTEN expression remained essentially unchanged (Fig. 4B). These results revealed that GGTI-298 may have inhibited pAKT signaling primarily through regulating pEGFR expression. Further studies were then conducted to elucidate how GGTI-298 mediates the downregulation of pEGFR. GGTI-298, a GGT1 inhibitor, prevents the geranylgeranylation of small G proteins such as RhoA (19), one of the most intensely studied proteins within the Rho family of GTPases, which is involved in various signaling regulations. Thus, it was hypothesized that GGTI-298 may have inhibited pEGFR through RhoA. Firstly, the present study detected the expression of RhoA. As shown in Fig. 4C, total RhoA increased in A549 cells following treatment with $5 \mu \mathrm{M}$ GGTI-298 when compared with the untreated

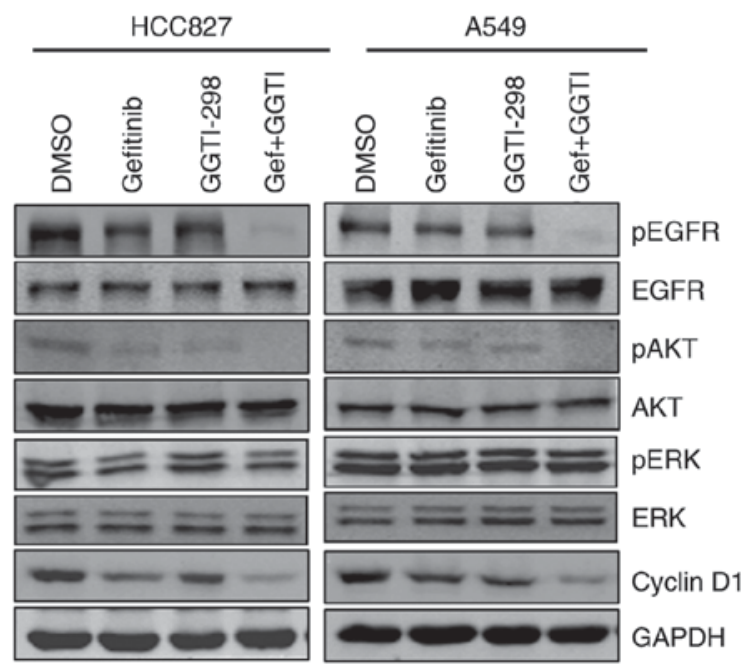

Figure 3. Effects of gefitinib and GGTI-298 on the EGFR signaling pathway and cyclin D1. Protein levels for the EGFR signaling pathway and cyclin D1 were measured by immunoblotting analysis. HCC 827 and A549 cells were treated with DMSO, gefitinib (HCC827, $20 \mathrm{nM}$; A549, $5 \mu \mathrm{M}$ ), $5 \mu$ M GGTI-298 or a combination of gefitinib and GGTI-298 for $48 \mathrm{~h}$. Representative results are shown ( $\mathrm{n}=3$ biological replicates). GGTI/GGTI-298, geranylgeranyl transferase 1 inhibitor; Gef, gefitinib; DMSO, dimethylsulfoxide; p-, phosphorylated; EGFR, epidermal growth factor receptor; AKT, protein kinase B; ERK, extracellular signal-regulated kinase.

(DMSO) group. Then, pull-down assays were conducted to detect active RhoA, which is responsible for the bioactivity of RhoA (21). Compared with control cells, GGTI-298 markedly impaired RhoA activation (Fig. 4C). These results indicated 


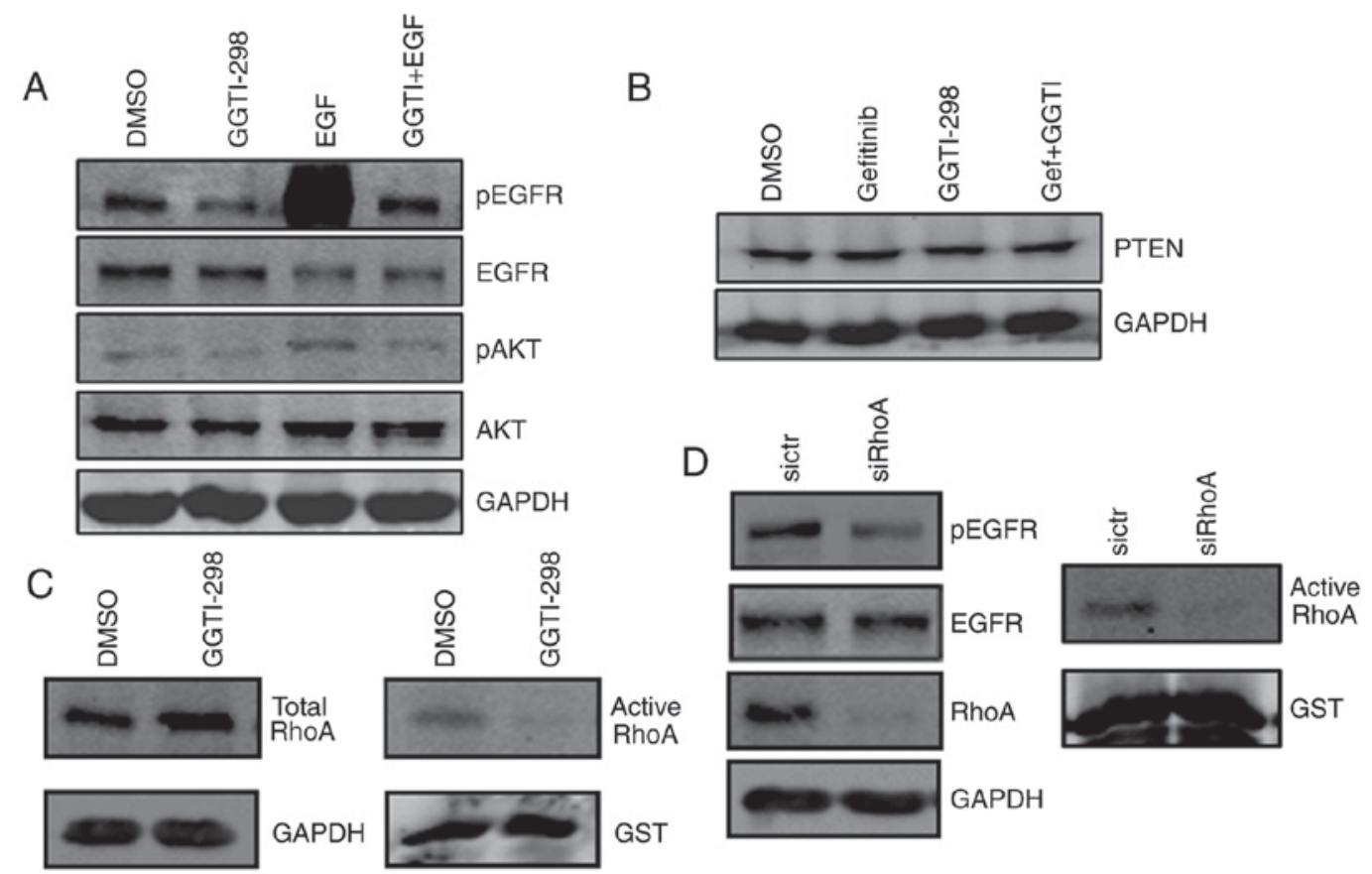

Figure 4. GGTI-298 inhibits the EGFR-AKT signaling pathway through RhoA. (A) GGTI-298 inhibited the phosphorylation of AKT and EGFR induced by EGF. A549 cells were treated with $5 \mu \mathrm{M}$ GGTI-298 in serum-free medium for $48 \mathrm{~h}$. Prior to protein extraction, cultures were exposed to $30 \mathrm{ng}$ EGF for $30 \mathrm{~min}$, as indicated in the figure. (B) PTEN levels were measured by immunoblotting analysis. A549 cells were treated with DMSO, $5 \mu \mathrm{M}$ gefitinib, $5 \mu \mathrm{M}$ GGTI-298 or a combination of gefitinib and GGTI-298 for $48 \mathrm{~h}$. GAPDH was used as an internal control. (C) Activity of RhoA was measured in A549 cells treated with DMSO and GGTI-298 for 48 h. (D) A549 cells were transfected with control siRNA or siRhoA, and the activity of RhoA and the phosphorylation of EGFR were measured $72 \mathrm{~h}$ following transfection. GST was used as a control in the pull-down assays. Representative results are shown (n=3 biological replicates). GGTI/GGTI-298, geranylgeranyl transferase 1 inhibitor; Gef, gefitinib; DMSO, dimethylsulfoxide; p-, phosphorylated; EGFR, epidermal growth factor receptor; AKT, protein kinase B; PTEN, phosphatase and tensin homolog; RhoA, Ras homolog family member A; si-/siRNA, small interfering RNA; GST, glutathione S-transferase.

that GGTI-298 inhibited RhoA activation in A549 cells. To detect the involvement of the RhoA protein in EGFR signaling, cells were transfected with siRNAs for RNA interference and the effects were analyzed. As detected by immunoblotting, the transfection of RhoA-specific siRNA resulted in the marked inhibition of total and active RhoA expression, and the downregulation of pEGFR (Fig. 4D). Taken together, these results validated the critical role of RhoA in the regulation of pEGFR and suggested that GGTI-298 may inhibit pEGFR signaling primarily through RhoA in A549 cells.

\section{Discussion}

Treatment of advanced NSCLC was revolutionized by EGFR TKIs such as gefitinib and erlotinib. Inadequate inhibition of EGFR signaling is considered to significantly contribute to TKI resistance and disease progression (22). Thus, effective inhibition of EGFR function through multiple mechanisms may augment EGFR TKI activity and overcome TKI resistance. The combination therapy consisting of afatinib and cetuximab previously presented promising clinical efficacy (22), indicating that the co-inhibition of EGFR may a potential method for enhancing the antitumor response. The present study revealed that GGTI-298, a GGT1 inhibitor, inhibited the EGFR signaling pathway mainly through RhoA, and a combined treatment of GGTI-298 and gefitinib impaired the phosphorylation of EGFR more thoroughly than either compound alone, significantly inhibiting cell proliferation and migration, and promoting apoptosis.

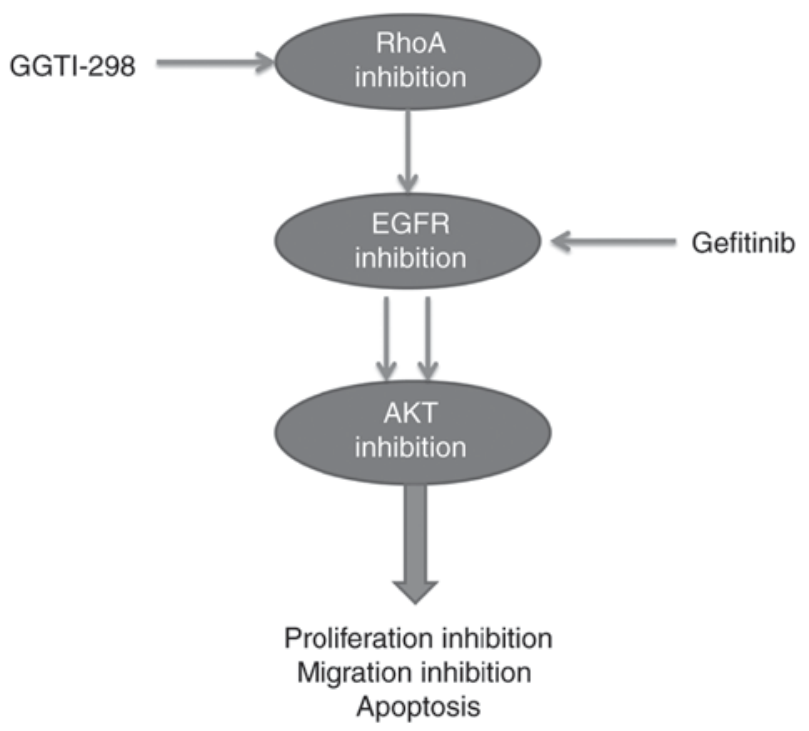

Figure 5. Schematic representation of the effect of the combined treatment of gefitinib and GGTI-298 on the inhibition of EGFR signaling. In vitro combination treatments of gefitinib and GGTI-298 inhibited the EGFR signaling pathway more thoroughly than either agent alone, resulting in the inhibition of cell proliferation and migration, and the promotion of apoptosis. GGTI-298, geranylgeranyl transferase 1 inhibitor; RhoA, Ras homolog family member A; EGFR, epidermal growth factor receptor; AKT, protein kinase B.

EGFR signaling is regulated by multiple protein molecules (23-26). In the present study, the EGFR-AKT signaling 
pathway was inhibited by the GGT1 inhibitor GGTI-298. Further experiments demonstrated that there was a slight upregulation of total RhoA expression following GGTI-298 treatment. However, the expression of active RhoA was markedly inhibited by GGTI-298. These results indicated that GGTI-298 inhibited RhoA activation. Crosstalk involving RhoA and other proteins has been widely reported $(14,27,28)$. In the present study, transfection experiments with siRNA against RhoA decreased the level of pEGFR. A study has previously demonstrated that actin organization served an important role in EGFR localization and activation (29), and the bioactivity of RhoA was critical for actin organization (30), which further supports the present study's results regarding the effect of RhoA on EGFR signaling. Taken together, these results indicated that GGTI-298 may inhibit the EGFR-AKT signaling pathway mainly through RhoA in A549 cells.

AKT and ERK are two main downstream signaling proteins of the EGFR signaling pathway (31). In the present study, the expression of pAKT was markedly decreased following GGTI-298 treatment. Mechanistic studies further revealed that GGTI-298 inhibited the EGFR activation induced by EGF; however, it had little effect on PTEN expression, which negatively regulates pAKT expression. These results suggested that GGTI-298 inhibited pAKT through EGFR, though not PTEN. However, the expression of pERK was essentially unchanged following treatment with GGTI-298. It was hypothesized that Ras, which is upstream of pERK, may mediate activity mainly through farnesylation, but not geranylgeranylation, which is consistent with the results of Sebti et al (32).

Recent studies have demonstrated the potential for mevalonate metabolite depletion to affect EGFR activity $(33,34)$. Targeting the mevalonate pathway inhibited the function of EGFR (35). Combinations of statins, inhibitors of the mevalonate pathway, and an EGFR inhibitor, such as gefitinib and cetuximab, induced a potent synthetical cytotoxicity $(31,36)$. However, it should be noted that statins inhibit the geranylgeranylation of RhoA as well as the synthesis of many other types of bioactive substances such as cholesterol and ubiquinone (19). Cholesterol is a critical component for the structure of animal cell membranes (37), and ubiquinone participates in aerobic cellular respiration (38). Thus, the use of statins may cause multiple side effects associated with these bioactive substances. In the present study, the results revealed that the inhibition of RhoA geranylgeranylation by GGTI-298 also suppressed the EGFR-AKT signaling pathway, and it was not involved in the synthesis of cholesterol and ubiquinone (19). Upon combination treatment with GGTI-298 and gefitinib, the synthetical loss of EGFR-AKT activation in A549 and HCC 827 cells resulted in a marked increase in the inhibition of cell proliferation and migration inhibition, and increased levels of apoptosis (Fig. 5). The effect of the GGTI alone was not satisfactory (39), which indicated that a combination-based therapy with a GGTI and other agents may be a potential option for augmenting the antitumor efficiency of GGTIs. Thus, these results were suggestive of the potential of combining these two drugs targeting EGFR, through distinct mechanisms; they therefore represent a novel, promising therapeutic strategy in lung cancer.
In conclusion, the results of the present study suggested that the antitumor efficacy could be markedly increased by combining gefitinib with GGTI-298, a specific inhibitor of protein geranylgeranylation. However, further investigation is required to evaluate the clinical efficacy of this combinatorial treatment in lung cancer.

\section{Acknowledgements}

Not applicable.

\section{Funding}

The present study was supported by the National Natural Science Foundation of China (grant no. 81621003), the National Key R\&D Program of China (grant nos. 2016YFC1303200 and 2016YFC1303203) and the National Natural Science Foundation of China (grant no. 81572731).

\section{Availability of data and materials}

The datasets used and/or analyzed during the current study are available from the corresponding author on reasonable request.

\section{Authors' contributions}

BSL and XYD designed and performed experiments, analyzed data and prepared the manuscript. HWX, HJX and QLT conducted the experiments associated with Figs. 1 and 2. QYG and YZN analyzed the data and contributed to the manuscript. FB supervised these experiments, analyzed data, and prepared the manuscript. All authors reviewed the final manuscript.

\section{Ethics approval and consent to participate}

Not applicable.

\section{Consent for publication}

Not applicable.

\section{Competing interests}

The authors declare that they have no competing interests.

\section{References}

1. Mok TS, Wu YL, Thongprasert S, Yang CH, Chu DT, Saijo N, Sunpaweravong P, Han B, Margono B, Ichinose Y, et al: Gefitinib or carboplatin-paclitaxel in pulmonary adenocarcinoma. N Engl J Med 361: 947-957, 2009.

2. Maemondo M, Inoue A, Kobayashi K, Sugawara S, Oizumi S, Isobe $\mathrm{H}$, Gemma A, Harada M, Yoshizawa H, Kinoshita I, et al: Gefitinib or chemotherapy for non-small-cell lung cancer with mutated EGFR. N Engl J Med 362: 2380-2388, 2010.

3. Zhou C, Wu YL, Chen G, Feng J, Liu XQ, Wang C, Zhang S, Wang J, Zhou S, Ren S, et al: Erlotinib versus chemotherapy as first-line treatment for patients with advanced EGFR mutation-positive non-small-cell lung cancer (OPTIMAL, CTONG-0802): A multicentre, open-label, randomised, phase 3 study. Lancet Oncol 12: 735-742, 2011.

4. Nicholson RI, Gee JM and Harper ME: EGFR and cancer prognosis. Eur J Cancer 37 (Suppl 4): S9-S15, 2001. 
5. Kobayashi S, Boggon TJ, Dayaram T, Jänne PA, Kocher O, Meyerson M, Johnson BE, Eck MJ, Tenen DG and Halmos B: EGFR mutation and resistance of non-small-cell lung cancer to gefitinib. N Engl J Med 352: 786-792, 2005.

6. Yokoyama K, Goodwin GW, Ghomashchi F, Glomset JA and Gelb MH: A protein geranylgeranyltransferase from bovine brain: Implications for protein prenylation specificity. Proc Natl Acad Sci USA 88: 5302-5306, 1991.

7. Reid TS, Terry KL, Casey PJ and Beese LS: Crystallographic analysis of CaaX prenyltransferases complexed with substrates defines rules of protein substrate selectivity. J Mol Biol 343 417-433, 2004.

8. Kusama T, Mukai M, Tatsuta M, Matsumoto Y, Nakamura H and Inoue M: Selective inhibition of cancer cell invasion by a geranylgeranyltransferase-I inhibitor. Clin Exp Metastasis 20: 561-567, 2003

9. Sun J, Qian Y, Chen Z, Marfurt J, Hamilton AD and Sebti SM: The geranylgeranyltransferase I inhibitor GGTI-298 induces hypophosphorylation of retinoblastoma and partner switching of cyclin-dependent kinase inhibitors. A potential mechanism for GGTI-298 antitumor activity. J Biol Chem 274: 6930-6934, 1999.

10. Dan HC, Jiang K, Coppola D, Hamilton A, Nicosia SV, Sebti SM and Cheng JQ: Phosphatidylinositol-3-OH kinase/AKT and survivin pathways as critical targets for geranylgeranyltransferase I inhibitor-induced apoptosis. Oncogene 23: 706-715, 2004.

11. Sonnemann J, Bumbul B and Beck JF: Synergistic activity of the histone deacetylase inhibitor suberoylanilide hydroxamic acid and the bisphosphonate zoledronic acid against prostate cancer cells in vitro. Mol Cancer Ther 6: 2976-2984, 2007.

12. Berndt N, Hamilton AD and Sebti SM: Targeting protein prenylation for cancer therapy. Nat Rev Cancer 11: 775-791, 2011.

13. McGuire TF, Qian Y, Vogt A, Hamilton AD and Sebti SM: Platelet-derived growth factor receptor tyrosine phosphorylation requires protein geranylgeranylation but not farnesylation. J Biol Chem 271: 27402-27407, 1996.

14. Li F, Jiang Q, Shi KJ, Luo H, Yang Y and Xu CM: RhoA modulates functional and physical interaction between ROCK1 and Erk1/2 in selenite-induced apoptosis of leukaemia cells. Cell Death Dis 4: e708, 2013.

15. Del Re DP, Miyamoto S and Brown JH: Focal adhesion kinase as a RhoA-activable signaling scaffold mediating Akt activation and cardiomyocyte protection. J Biol Chem 283: 35622-35629, 2008.

16. Basile JR, Gavard J and Gutkind JS: Plexin-B1 utilizes RhoA and Rho kinase to promote the integrin-dependent activation of Akt and ERK and endothelial cell motility. J Biol Chem 282: 34888-34895, 2007

17. Hebert M, Potin S, Sebbagh M, Bertoglio J, Breard J and Hamelin J: Rho-ROCK-dependent ezrin-radixin-moesin phosphorylation regulates Fas-mediated apoptosis in Jurkat cells J Immunol 181: 5963-5973, 2008.

18. Chou TC, Motzer RJ, Tong Y and Bosl GJ: Computerized quantitation of synergism and antagonism of taxol, topotecan, and cisplatin against human teratocarcinoma cell growth: A rational approach to clinical protocol design. J Natl Cancer Inst 86: $1517-1524,1994$

19. Sorrentino G, Ruggeri N, Specchia V, Cordenonsi M, Mano M, Dupont S, Manfrin A, Ingallina E, Sommaggio R, Piazza S, et al: Metabolic control of YAP and TAZ by the mevalonate pathway. Nat Cell Biol 16: 357-366, 2014.

20. Li P, Wei J and Gao X: Insulin promotes the proliferation of human umbilical cord matrix-derived mesenchymal stem cells by activating the Akt-Cyclin D1 axis. Stem Cells Int 2017: 7371615, 2017

21. Deng W, Gu L, Li X, Zheng J, Zhang Y, Duan B, Cui J, Dong J and Du J: CD24 associates with EGFR and supports EGF/EGFR signaling via RhoA in gastric cancer cells. J Transl Med 14: 32 , 2016.
22. Janjigian YY, Smit EF, Groen HJ, Horn L, Gettinger S, Camidge DR, Riely GJ, Wang B, Fu Y, Chand VK, et al: Dual inhibition of EGFR with afatinib and cetuximab in kinase inhibitor-resistant EGFR-mutant lung cancer with and without T790M mutations. Cancer Discov 4: 1036-1045, 2014.

23. Fischer OM, Hart S, Gschwind A and Ullrich A: EGFR signal transactivation in cancer cells. Biochem Soc Trans 31: 1203-1208, 2003.

24. Song S, Honjo S, Jin J, Chang SS, Scott AW, Chen Q, Kalhor N, Correa AM, Hofstetter WL, Albarracin CT, et al: The Hippo coactivator YAP1 mediates EGFR overexpression and confers chemoresistance in esophageal cancer. Clin Cancer Res 21: 2580-2590, 2015.

25. He C, Mao D, Hua G, Lv X, Chen X, Angeletti PC, Dong J, Remmenga SW, Rodabaugh KJ, Zhou J, et al: The Hippo/YAP pathway interacts with EGFR signaling and HPV oncoproteins to regulate cervical cancer progression. EMBO Mol Med 7: $1426-1449,2015$

26. Chrysogelos SA and Dickson RB: EGF receptor expression, regulation, and function in breast cancer. Breast Cancer Res Treat 29: 29-40, 1994

27. Zhang Y, Xia H, Ge X, Chen Q, Yuan D, Chen Q, Leng W, Chen L, Tang Q and Bi F: CD44 acts through RhoA to regulate YAP signaling. Cell Signal 26: 2504-2513, 2014.

28. Li H, Ung CY, Ma XH, Li BW, Low BC, Cao ZW and Chen YZ: Simulation of crosstalk between small GTPase RhoA and EGFR-ERK signaling pathway via MEKK1. Bioinformatics 25: 358-364, 2009

29. Lunn JA, Wong H, Rozengurt E and Walsh JH: Requirement of cortical actin organization for bombesin, endothelin, and EGF receptor internalization. Am J Physiol Cell Physiol 279: C2019-C2027, 2000

30. Asanuma K, Yanagida-Asanuma E, Faul C, Tomino Y, Kim K and Mundel P: Synaptopodin orchestrates actin organization and cell motility via regulation of RhoA signalling. Nat Cell Biol 8: 485-491, 2006.

31. Lee J, Lee I, Han B, Park JO, Jang J, Park C and Kang WK: Effect of simvastatin on cetuximab resistance in human colorectal cancer with KRAS mutations. J Natl Cancer Inst 103: 674-688, 2011.

32. Sebti SM and Hamilton AD: Farnesyltransferase and geranylgeranyltransferase I inhibitors and cancer therapy: Lessons from mechanism and bench-to-bedside translational studies. Oncogene 19: 6584-6593, 2000.

33. Mantha AJ, Hanson JE, Goss G, Lagarde AE, Lorimer IA and Dimitroulakos J: Targeting the mevalonate pathway inhibits the function of the epidermal growth factor receptor. Clin Cancer Res 11: 2398-2407, 2005.

34. Niknejad N, Morley M and Dimitroulakos J: Activation of the integrated stress response regulates lovastatin-induced apoptosis. J Biol Chem 282: 29748-29756, 2007.

35. Dimitroulakos J, Lorimer IA and Goss G: Strategies to enhance epidermal growth factor inhibition: Targeting the mevalonate pathway. Clin Cancer Res 12: 4426s-4431s, 2006.

36. Hwang KE, Kwon SJ, Kim YS, Park DS, Kim BR, Yoon KH, Jeong ET and Kim HR: Effect of simvastatin on the resistance to EGFR tyrosine kinase inhibitors in a non-small cell lung cancer with the T790M mutation of EGFR. Exp Cell Res 323: 288-296, 2014.

37. Simons K and Sampaio JL: Membrane organization and lipid rafts. Cold Spring Harb Perspect Biol 3: a004697, 2011.

38. Ernster L and Dallner G: Biochemical, physiological and medical aspects of ubiquinone function. Biochim Biophys Acta 1271: 195-204, 1995

39. Lobell RB, Liu D, Buser CA, Davide JP, DePuy E, Hamilton K, Koblan KS, Lee Y, Mosser S, Motzel SL, et al: Preclinical and clinical pharmacodynamic assessment of L-778,123, a dual inhibitor of farnesyl:protein transferase and geranylgeranyl:protein transferase type-I. Mol Cancer Ther 1: 747-758, 2002. 